\title{
A Hybrid Continuum / Particle Approach for Micro-Scale Gas Flows
}

\author{
Quanhua Sun*, Iain D. Boyd" and Graham V. Candler ${ }^{\dagger}$ \\ "Department of Aerospace Engineering, University of Michigan, Ann Arbor, MI 48109 \\ ${ }^{\dagger}$ Department of Aerospace Engineering and Mechanics, University of Minnesota, Minneapolis, MN 55455
}

\begin{abstract}
A hybrid continuum/particle approach is proposed for micro scale gas flows in this paper. The approach couples the DSMC-IP method and a Navier-Stokes solver with an adaptive interface. The continuum solver uses the particle cells as ghost cells because the IP method preserves the hydrodynamic information that the continuum solver uses. In order to generate particles from the continuum side, two strategies are proposed. The first one uses a condition similar to the Marshak condition in generating particles through the interface. The second strategy adopts buffer cells and reservoir cells, which avoids directly generating particles. The interface is determined by a continuum breakdown parameter that is evaluated in every time step. In order to track the interface, a mapping technique is used in the code. Numerical examples show that the hybrid approach couples the continuum solver and the particle method very smoothly. Simulated results also show the effects of the cutoff value of the continuum breakdown parameter.
\end{abstract}

\section{INTRODUCTION}

Micro scale gas flows [1] prevail in many micro-electro-mechanical systems (MEMS) [2], such as microturbines, gas chromatographs, and micro air vehicles (MAVs). Correct prediction of these flows is important to the design and development of MEMS. However, simulation of such flows is very complicated because the small characteristic length of MEMS is comparable to the mean free path of the gas molecules, which means the continuum equations are no longer valid and solution of the Boltzmann equation must be considered.

The Boltzmann equation describes the time evolution of a distribution function of microscopic particles. Methods based on this equation are generally numerically expensive especially when flows progress from free molecular, through transitional, to continuum regions. As micro scale gas flows are usually subsonic, modeling these flows involves a domain that is much larger than the system itself, which means that most of the domain can be described by the continuum equations. Since continuum solvers are several orders of magnitude more effective than molecular dynamics (MD) techniques or the direct simulation Monte Carlo (DSMC) method [3], an effective approach for micro scale gas flows is to combine the accuracy of kinetic methods and the efficiency of continuum solvers. Namely, hybrid techniques reduce the computational cost of a numerical simulation by limiting particle methods to the regions where the kinetic equations must be applied, and use continuum methods in the majority of the computational domain.

In the past ten years, many works have been reported with reference to hybrid techniques. There exist weakly coupled schemes $[4,5]$ for which a continuum solver provides a boundary condition for a particle method. There are also overlapping coupling strategies for which a particle method provides a boundary condition for a continuum scheme [6], or even provides transport coefficients for a continuum method [7]. However, strongly non-overlapping coupled schemes may give better performance for accuracy and speed. Wadsworth and Erwin first demonstrated such a scheme by simulating one-dimensional shock waves [8] and two-dimensional slit flows [9] using a property extrapolation technique. Hash and Hassan [10] performed detailed studies of a hybrid code and suggested that the Mashak condition is a better coupling technique. However, in a later paper [11], they pointed out that the large statistical scatter from the DSMC method precluded the application of the Mashak condition to low speed flows. Related coupling schemes are also available in the literature $[12,13,14,15,16]$. For further development of hybrid methods, adaptive hybrid schemes are therefore proposed, especially for unsteady rarefied flows. Roveda et al. [17] described a Euler/particle approach that can analyze unsteady flows by coupling an adaptive discrete velocity 
(ADV) Euler solver and the DSMC method with an adaptive interface. In a following paper [18], they successfully simulated an unsteady pressure driven slit flow with the scheme. There are other adaptive hybrid schemes, such as the adaptive domain decomposition method proposed by Tiwari and Klar [19], and the adaptive mesh and algorithm refinement (AMAR) method developed by Garcia et al [20].

However, most of the hybrid schemes exhibit scatter difficulty in the particle methods. Sampling a large number of particles is generally adopted by most authors with multiple time steps [8,10,16], ghost cells [17], or local average from multiple cells [14]. Then a large sampling size is required for low speed flows, which can be numerically expensive to couple two methods for each time step. In some cases, robust continuum solvers may circumvent this difficulty by some degree [16]. For general micro scale gas flows, however, a kinetic method with small statistical scatter must be coupled with a continuum solver in a hybrid approach.

In this paper, we efficiently couple a Navier-Stokes solver with the DSMC-IP method by a movable particle/continuum interface for each time step. The DSMC-IP method and the Navier-Stokes solver are summarized in the next section. In section 3, the hybrid approach is detailed. Numerical examples are then provided. Finally, we end with some concluding remarks.

\section{NUMERICAL METHODS}

\section{Kinetic Approach: the DSMC-IP method}

The Boltzmann equation of gas kinetic theory is very complicated due to the collision term involved. It is still numerically too expensive to use molecular dynamics following all the microscopic particles for most practical problems. In many cases, the direct simulation Monte Carlo method developed by Bird [3] is used for simulating rarefied gas flows as it is about six orders of magnitude faster than the molecular dynamics although it is much more expensive compared to traditional continuum solvers [13].

The DSMC method models a real gas flow with representative particles in simulated physical space. The position coordinates and the velocity components (even internal energy state) of simulated particles are preserved and are modified with time as the particles are concurrently followed through representative collisions and boundary interactions. Numerous simulations have shown the validity of the DSMC method for gases with short range binary interaction. However, the inherent statistical scatter from the method requires a large sampling size, which prevents its application for general low speed micro scale gas flows.

Aimed to reduce the statistical scatter in the DSMC method, an information preservation (IP) method [21,22] was recently developed based on the DSMC method. The IP method reduces the statistical scatter by preserving macroscopic information of the flow in the particles and in the computational cells simulated in the DSMC method. The preserved macroscopic information of particles is updated during collisions as well as the microscopic information, and is then modified to include the pressure field effects excluded in the collisions. Therefore the simulated particles in one cell may have different preserved macroscopic information since the particles come from different locations following their microscopic movements. An additional energy transfer model and a collision model were proposed in reference 22 , and the examples showed the validity of the IP method for typical low speed steady flows and low frequency unsteady flows ranging from the near-continuum regime to the free molecular regime. Hence, the IP method is a kinetic method.

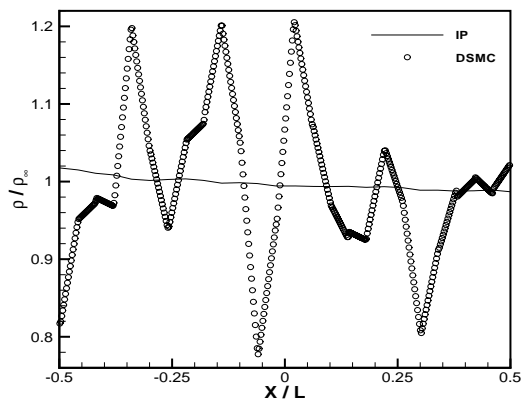

(a) Sampling size: 20

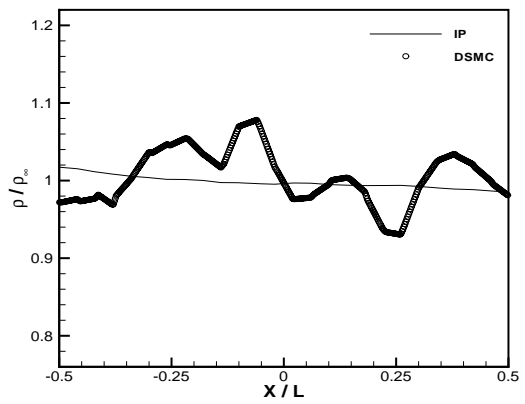

(b) Sampling size: 20,000

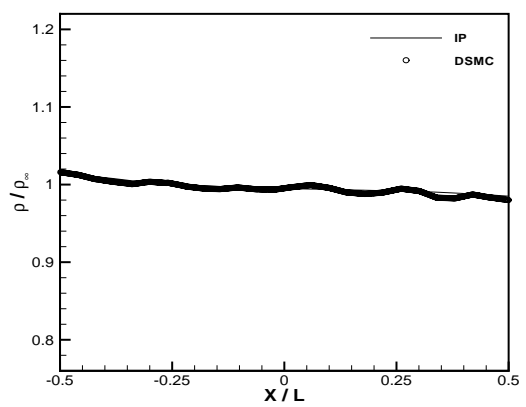

(c) Sampling size: $20,000,000$

FIGURE 1. Comparison of Statistical Scatter from the IP Method and the DSMC Method. 
The main benefit of the IP method is its rather small statistical scatter compared with the DSMC method for micro scale gas flows, which can greatly reduce the computational cost for these flows. Boundary treatments used in computational fluid dynamics [23] can be used for the IP method, which circumvents some difficulty of the boundary conditions in the DSMC method [24, 25]. Also, development of an adaptive hybrid scheme for micro scale gas flows becomes possible. Figure 1 illustrates the statistical scatter of the IP and the DSMC method by showing the density profile along a straight line from a low speed flow over a flat plate.

\section{The Navier-Stokes Solver}

Compared with particle methods, computational fluid dynamics is well developed. In our hybrid approach, the continuum approach solves the compressible Navier-Stokes equations using a finite volume formation. The fluxes are evaluated with a second-order accurate modified Steger-Warning flux-vector splitting approach [26]. In order to extend the validity of the Navier-Stokes equations, slip wall boundary conditions are implemented with the use of the Maxwell-type slip velocity expression. Characteristic boundary conditions [23] are used for open boundaries.

\section{THE HYBRID APPROACH}

In our adaptive hybrid approach, the IP method acts as the kinetic method while the Navier Stokes solver is used in the continuum domain. Both solvers are implemented in the MONACO system, which was first developed by Dietrich and Boyd as a DSMC code [27]. The continuum domain and the IP domain are separated by an interface. Through the interface, the continuum domain and the IP domain exchange information in every time step. The interface is time adaptive, and its location is determined by a continuum breakdown parameter.

\section{Coupling the IP method and the Navier-Stokes Solver}

The IP method and the continuum solver are coupled with an interface. The particle domain needs the interface to generate particles while the continuum solver requires fluxes through the interface. Therefore, the interface acts as a boundary for each domain. For general hybrid schemes, the boundary condition for the continuum solver is very difficult or expensive to obtain because of the large statistical scatter in particle methods. However, the IP method preserves the hydrodynamic information in cells that the Navier-Stokes solver uses. The interface for the continuum solver is then totally internal because the continuum solver can use the IP cells as continuum cells and evaluate the fluxes through the interface. Generally the very small statistical scatter in the IP region will not cause instability of the Navier-Stokes solver.

For hybrid approaches, particles entering the continuum domain are removed. However, there are particles that enter the particle domain from the continuum side for subsonic flows. In most hybrid schemes, the number of entering particles and their microscopic information are determined from the half fluxes based on the hydrodynamic values on the interface. The velocity of the particles is sampled from a Maxwellian distribution, which is only valid for equilibrium flows. For a hybrid scheme, a large continuum domain is obviously desired as the continuum solver is more numerically efficient. Hence, the interface will be located in a near-equilibrium region where a velocity gradient or temperature gradient may exist. For this reason, it was suggested [10] that a Chapman-Enskog distribution (Equations 1-4) [28] must be used. It may also not be accurate to calculate the half fluxes from the continuum side simply based on the hydrodynamic values on the interface. Garcia et al. [20] used the macroscopic values on the interface and their gradients on the nearby continuum cells to generate the particles. In our hybrid approach, we use two different strategies to deal with the half fluxes from the continuum side.

$$
\begin{gathered}
f(\vec{C})=f_{0}(\vec{C}) \Gamma(\vec{C}), \vec{C}=\vec{c} /(2 k T / m)^{1 / 2} \\
f_{0}(\vec{C})=\frac{1}{\pi^{3 / 2}} e^{-C^{2}} \\
\Gamma(\vec{C})=1+q_{i}^{*} C_{i}\left(\frac{2}{5} C^{2}-1\right)-\tau_{i j}^{*} C_{i} C_{j} \\
q_{i}^{*}=-\frac{\kappa}{P}\left(\frac{2 m}{k T}\right)^{1 / 2} \frac{\partial T}{\partial x_{i}}, \tau_{i j}^{*}=\frac{\mu}{P}\left(\frac{\partial v_{i}}{\partial x_{j}}+\frac{\partial v_{j}}{\partial x_{i}}-\frac{2}{3} \frac{\partial v_{k}}{\partial x_{k}} \delta_{i j}\right)
\end{gathered}
$$

A condition similar to the Marshak condition is used in our first strategy. The full fluxes crossing the interface based on the local hydrodynamic values are set to be the sum of the counted half fluxes from the IP side and the half 
fluxes from the continuum side that will be determined. Equations 5-7 show this condition, where subscripts "p" and "c" represent the IP side and the continuum side, respectively. After the macroscopic information for the entering particles is determined, the number of entering particles is rounded to the nearest integer because particles cannot be generated as a fractional number. Then the difference of the half fluxes is stored and is added to the next time step ensuring that the half fluxes from the continuum side are implemented. As can be seen, all new particles have the same macroscopic information, while the microscopic information of the particles is sampled from the ChapmanEnskog distribution based on the local hydrodynamic values [28]. However, numerical examples (not shown here) indicate that small velocity scatter in the particles from the IP side may cause large temperature scatter in new particles for high-speed flows because fluctuation of the translational energy affects internal energy through the total energy (compared with low speed flows, high speed flows have larger variation of the translational energy for the same level variation of the velocity), which may crash the code for simulation. For isothermal gas flows, however, this is not a problem. Therefore, this strategy may be reserved for isothermal flows. For the rest of the paper, this strategy is not used unless specified.

$$
\begin{gathered}
N_{c}+N_{p}=\frac{f_{\rho} \cdot A \cdot d t}{m} \\
N_{c} \vec{c}_{c}+N_{p} \sum \vec{v}_{p, j}=\frac{\left(\vec{f}_{\rho \bar{v}}-p \vec{n}\right) \cdot A \cdot d t}{m} \\
N_{c}\left(e+0.5 v^{2}\right)_{c}+N_{p} \sum\left(e+0.5 v^{2}\right)_{p, j}=\frac{\left(f_{\rho\left(e+0.5 v^{2}\right)}-p \vec{v} \cdot \vec{n}\right) \cdot A \cdot d t}{m}
\end{gathered}
$$

A second strategy is developed to avoid directly generating particles on the interface. Buffer cells and reservoir cells are used in the continuum domain as illustrated in Figure 2. These buffer cells and reservoir cells are also treated as particle cells for particle movement and particle collisions. Then the interface becomes the internal cell edge for the IP treatment. The reservoir cells are used to generate particles that can enter the IP domain, which avoids directly generating particles on the interface. The buffer cells, however, improve the quality of the particles that enter the IP domain. Here is the algorithm for this strategy.

1) Generate all represented particles for the buffer cells according to the Chapman-Enskog distribution based on the local macroscopic information.

2) During each time step, generate particles for the reservoir cells according to the Chapman-Enskog distribution.

3) Particles in the buffer cells and reservoir cells are selected for collisions and move around using general IP procedures. The particles leaving particle cells (including buffer cells and reservoir cells) are removed.

4) Remove particles in the reservoir cells.

5) Repeat steps 2-4 until the simulation is finished.

Obviously, more buffer cells used for the interface will improve the interface properties. However, this will increase the computational task, which contradicts the intention of hybrid schemes. Hence, only one or several levels of buffer cells are used. The number of reservoir cells is also determined such that a negligible number of particles will enter any buffer cell from a continuum cell during one time step. Generally, two or more levels of reservoir cells are required.

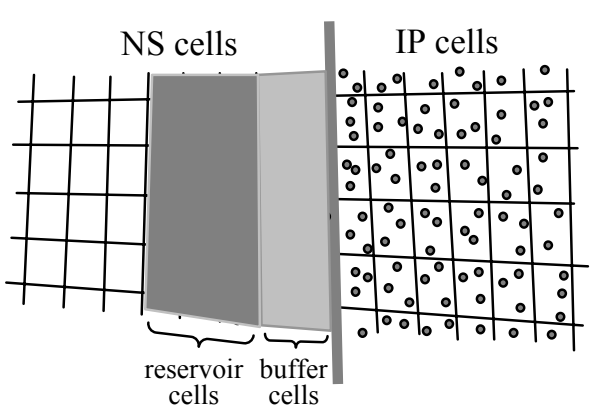

FIGURE 2. Interface and Cell Structures.

$\begin{array}{rrrrrrrrrrrrrrrrrrr}10 & 10 & 0 & 0 & 0 & 0 & 0 & 0 & 0 & 0 & 0 & 0 & 0 & 0 & 0 & 0 & 10 & 10 \\ 10 & 10 & 0 & 0 & 0 & 0 & 0 & 0 & 0 & 0 & 0 & 0 & 0 & 0 & 0 & 0 & 10 & 10 \\ 10 & 10 & 0 & 0 & 0 & 0 & 0 & 200 & 200 & 200 & 200 & 200 & 0 & 0 & 0 & 0 & 10 & 10 \\ 10 & 10 & 0 & 0 & 0 & 0 & 200 & 100 & 100 & 100 & 100 & 100 & 200 & 0 & 0 & 0 & 10 & 10 \\ 10 & 10 & 0 & 0 & 0 & 200 & 100 & 101 & 101 & 101 & 101 & 101 & 100 & 200 & 0 & 0 & 10 & 10 \\ 10 & 10 & 0 & 0 & 200 & 100 & 101 & 1 & 1 & 1 & 1 & 1 & 101 & 100 & 200 & 0 & 10 & 10 \\ 10 & 10 & 0 & 200 & 100 & 101 & 1 & 11 & 11 & 11 & 11 & 1 & 101 & 100 & 200 & 0 & 10 & 10 \\ 10 & 10 & 0 & 200 & 100 & 101 & 1 & 11 & 11 & 11 & 11 & 1 & 101 & 100 & 200 & 0 & 10 & 10 \\ 10 & 10 & 0 & 200 & 100 & 101 & 1 & 11 & 11 & 11 & 11 & 1 & 101 & 100 & 200 & 0 & 10 & 10\end{array}$

FIGURE 3. An Illustrative Map Showing Cell Types.

\section{Interface Determination}

Hybrid schemes can couple two methods by an interface because both methods are valid around the interface. In order to achieve the maximum benefit from a hybrid scheme, the interface should be placed in locations where 
continuum equations tend to breakdown. Hence, a continuum breakdown parameter should be adopted to determine the interface location. In the literature, several continuum breakdown parameters were proposed, including Bird's parameter $\mathrm{P}$ [29], the gradient-length local Knudsen number $\mathrm{Kn}_{\mathrm{GLL}}$ [30], Tiwari's criterion $\|\phi\|[31]$, and the parameter B by Garcia et al. [20]. However, these parameters have not been tested widely, and there is no cutoff value suggested for micro scale gas flows.

The Navier-Stokes equations can be derived from the Boltzmann equation using the Chapman-Enskog theory under near equilibrium conditions [32]. This analysis indicates that the Navier-Stokes equations are not valid when the nonlinear terms in the Chapman-Enskog expansion become important. In other words, the continuum equations break down when the velocity distribution function deviates from its equilibrium state by some degree. In fact, all the current parameters $[20,29-31]$ are a combination of the coefficients in the first order Chapman-Enskog expansion. Therefore, we select the breakdown parameter $B=\max \left\{\left|\tau_{i, j}^{*},\right|,\left|q_{i, j}^{*}\right|\right\}$ as our current particle/continuum interface indicator because this parameter includes all the coefficients in the first-order Chapman-Enskog expansion. However, other parameters can be implemented in the code without any difficulty.

There is no theory that shows the cutoff value that a specific continuum breakdown parameter should take. Some researchers investigate the cutoff value by computing the breakdown parameter when differences between a continuum solution and a kinetic result are larger than 5\% while some simply take a small number as the cutoff value. For subsonic flows, any difference between the continuum solution and kinetic result in one location will affect all of the computational domain. It is better to compare hybrid solutions with different cutoff values. Obviously, the cutoff value depends on the difference between the hybrid solution and the kinetic result that one can tolerate. In the results section, we provide an example to show the effects of cutoff value for the continuum breakdown parameter B.

\section{Interface Adaptation}

In many cases, the continuum/particle interface is unknown before the simulation or the interface changes with time. Hence, the interface needs to be relocated based on a given continuum breakdown parameter. A mapping technique is therefore used to track the interface. A similar technique can be found in reference 17.

Each computational cell is assigned a three-digit number to represent the type of cell, which can be a reservoir cell, a buffer cell, a continuum cell other than buffer cell or reservoir cell, or an IP cell. The number is in the form of $a+10 b+100 c$. Here, "a" indicates whether the cell is in the continuum domain $(0)$ or the IP domain (1). "b" shows whether the cell is adaptive (0) or not (1). This means that we can force some cells to be of a fixed type. "c" is used to indicate the levels of buffer cells or reservoir cells or to indicate the IP cells neighboring the interface. Figure 3 shows a simple map that illustrates the type of a cell.

The algorithm for setting the type of cell is as follows.

1) Sweep all computational cells to determine "a" by comparing the cutoff value of the continuum breakdown parameter to its local value. If the local value is smaller than the cutoff value, the cell is determined as a continuum cell $(\mathrm{a}=0)$. Otherwise, it is an IP cell $(\mathrm{a}=1)$. As can be seen, " $\mathrm{b}$ " generally does not change after it is initialized.

2) Set "c" to 1 for all cells neighboring the interface.

3) Set "c" to $i+1$ for the continuum cells neighboring buffer cells or reservoir cells whose "c" is $i(i>0)$ until all the buffer cells and reservoir cells are marked.

To end this section, we present the whole algorithm for the hybrid approach.

1) Initialize all necessary information for the simulation. Namely, first setup the computational cells and their type. Then distribute particles for the DSMC-IP cells (including buffer cells and reservoir cells) and assign hydrodynamic information for all computational cells.

During each time step, the following operations are executed.

2) All the particles move around and collide as in the usual IP code. When particles reach an open boundary or continuum cells (excluding the buffer cells and reservoir cells), they are removed. New particles are injected on the open boundary, but no particles are generated from the continuum cell side.

3) The hydrodynamic values of all cells are re-evaluated. All continuum cells (include buffer cells and reservoir cells) update their values according to the Navier-Stokes solver, while all IP cells sample the hydrodynamic information from the IP information of the particles contained in the cell.

4) Remove all particles in the reservoir cells.

5) Calculate the continuum breakdown parameter for every cell and use the interface adaptation algorithm to set new type for each cell. Then generate particles according to the Chapman-Enskog distribution for particle cells 
(including buffer cells and reservoir cells) where no particles exist, and remove particles from the continuum cells (excluding buffer cells and reservoir cells) that are occupied by particles. In many cases, it is not necessary to change the interface for every time step. Hence, a frequency for adapting the interface can also be implemented in the code.

\section{RESULTS}

We have applied the proposed hybrid approach for several simulations. In this section, simulation of a Couette flow is described using the first strategy of generating particles for the IP domain from the continuum side. We also simulate flows over micro scale objects. A flow over a flat plate at zero angle of attack is given as an example to show the ability of the hybrid approach and to reveal the effects of the cutoff value of the continuum breakdown parameter B.

\section{Couette Flow}

Couette flow is relatively simple because of its one dimensional property. Study of planar Couette flows under different rarefied conditions can be found in reference 22. Hash and Hassan [10] assessed a DSMC/NS hybrid scheme by simulating a planar Couette flow. We simulate this case to evaluate the first strategy for calculating the half fluxes from the continuum cells.

In the Couette flow, one of two parallel plates has a velocity of $300 \mathrm{~m} / \mathrm{s}$ while the other is at rest. The temperature of both plates is kept at $273 \mathrm{~K}$, and full momentum and thermal accommodation is assumed for both plates. The distance between the two plates and the nominal density of the flow are compatible such that the bodylength global Knudsen number of the flow is $0.01,0.03$, or 0.1 . In all three cases $\left(\mathrm{Kn}_{\mathrm{BLG}}=0.01,0.03\right.$, and 0.1$), 200$ computational cells are used, and the cell size is less than the mean free path of the Argon gas for which a variable hard sphere (VHS) molecular model [3] is used. The particle/continuum interface is fixed in three cases such that $70 \%$ of the cells are calculated by the Navier-Stokes solver (see Fig. 4). As mentioned earlier, in high speed flows, the velocity scatter can cause large temperature scatter, and then crash the code. Hence, the number of simulated particles in the IP cells is above 50 and is increased to about 500 when the Knudsen number is 0.1 .

Figure 4 shows the results from the hybrid approach, and comparison is made with the full IP results. When $\mathrm{Kn}_{\mathrm{BLG}}=0.01$, excellent agreement is obtained, which shows the consistency of the hybrid approach and the full IP method. The smooth results for the case with $\mathrm{Kn}_{\mathrm{BLG}}=0.03$ demonstrate that the interface is working well. The slight difference of the temperature profile in the continuum domain indicates that the continuum equations tend to be invalid for all the computational domain. When $\mathrm{Kn}_{\mathrm{BLG}}=0.1$, it shows the continuum equations are completely invalid and thus the interface cannot correctly pass the information. This also means it is impossible for the continuum equations with slip models to predict the flow.
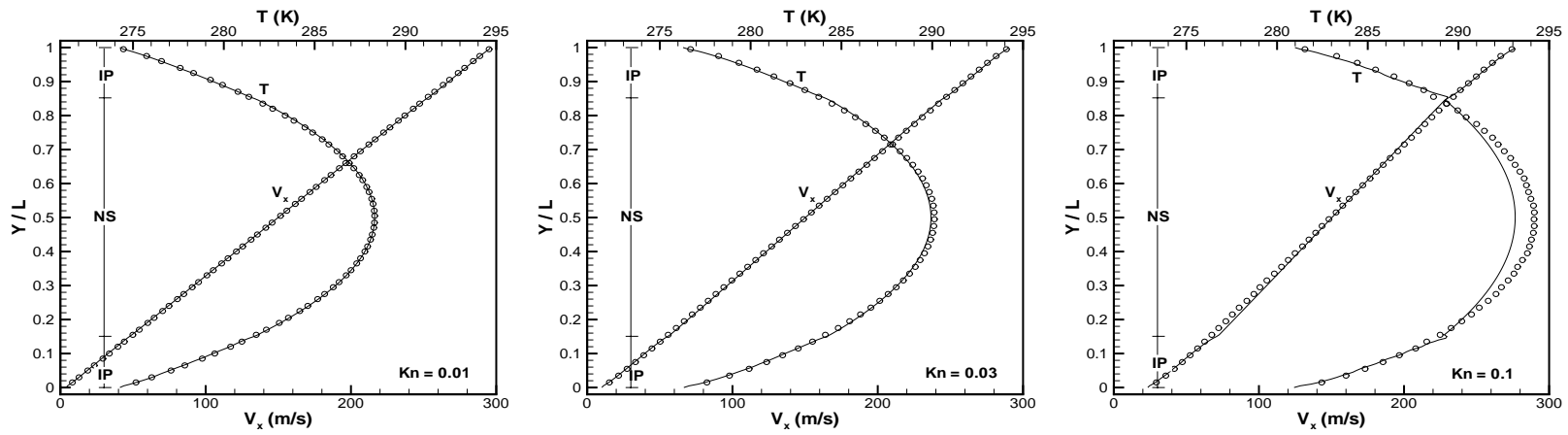

FIGURE 4. Comparison of Velocity and Temperature Distribution from the IP Code (circle) and the Hybrid Code (line).

The cutoff value of continuum breakdown parameters is essential to hybrid schemes. We compare profiles of different breakdown parameters for this simple case. The breakdown parameter $\mathrm{P}$ was proposed for expanding flows, and fails for this case as the mass density does not change along the streamline. The contribution of the local Mach number (or velocity) in the parameter $\mathrm{P}$ is also questionable for this case. As shown in Figure 5, the gradientlength local Knudsen also fails because this parameter is mainly zero (excluding the scatter) in the centerline of the Couette flow, while parameter $\|\phi\|$ and parameter B display similar reasonable behavior. 

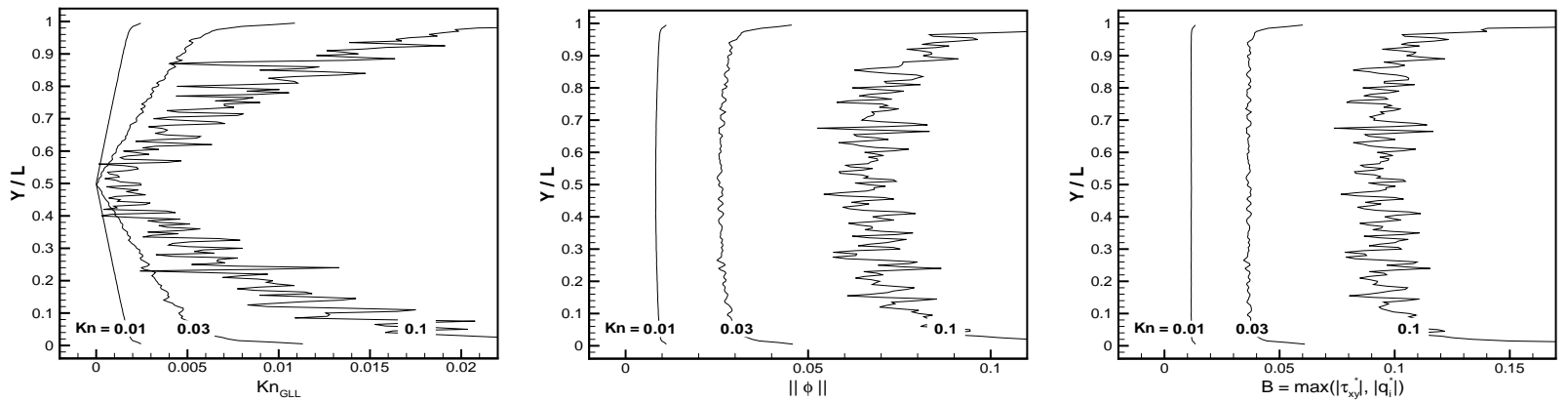

FIGURE 5. Profiles of Continuum Breakdown Parameters $\mathrm{Kn}_{\mathrm{GLL}},\|\phi\|$ and $\mathrm{B}$ for the Couette Flows under Different $\mathrm{Kn}_{\mathrm{BLG}}$ 's.

\section{Flow over a Flat Plate}

For subsonic external micro scale gas flows, a hybrid approach is no doubt the best choice for simulation because most of the computational domain can be described by the continuum equations. An airflow with a free stream Mach number of 0.2 over a 20 -micron-long flat plate is simulated here. The free stream temperature and the wall temperature are $295 \mathrm{~K}$, and the body-length global Knudsen number of the flow is about 0.024 (the Reynolds number is about 2.4). Full thermal accommodation is assumed in the simulation, and the VHS molecular model is used. A computation domain is set up with 60 microns in the upstream region, 130 microns in the downstream region and a full span of 120 microns. Characteristic boundary conditions are adopted. About 20 particles are used for every particle cell, and the time step is less than the mean collision time of the molecules.

Figure 6 shows the velocity profiles from the full IP method, the hybrid approach, and the Navier-Stokes solver. For the hybrid result in Fig. 6, the cutoff value of the parameter B is 0.005 , and the dashed line indicates the interface. The overall agreement of the results from three methods is good. This is not surprising because the flow is in the slip regime. Detailed comparison is presented in Fig. 7. The cutoff value of B is 0.002 for hybrid 1, 0.005 for hybrid 2, and 0.01 for hybrid 3. As can be seen, the surface pressure is very close for all shown results except the result from hybrid 3. It is found that the shear stress on the surface and the slip velocity decrease when the IP domain shrinks. The continuum solver predicts the surface pressure well, while it only gives fair shear stress and underpredicts the slip velocity. It seems 0.002 is an acceptable value of the continuum breakdown parameter B for this flow. Compared with the value in the Couette flow, the value of B is much smaller here. The reason is not clear yet. Simulations (not shown here) of a flow with Mach number of 0.1 and body-length global Knudsen number of 0.1 over a $5 \%$ plate with the angle of attack of $10^{\circ}$ indicates that 0.002 is also an acceptable value for the parameter B. However, more detailed investigation is required to establish a cutoff value for the parameter B.
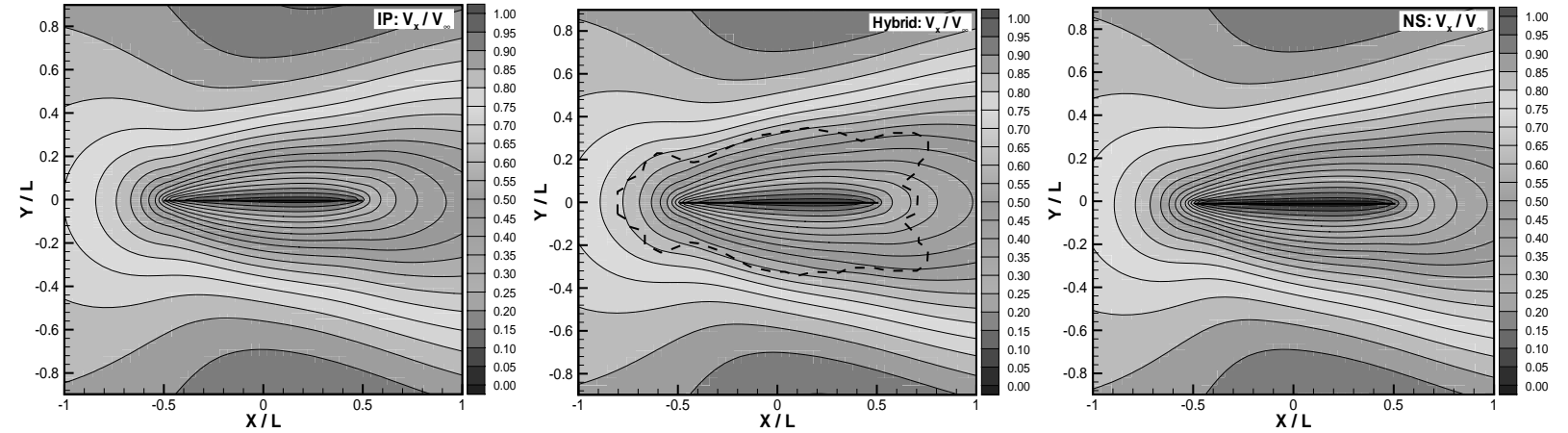

FIGURE 6. Comparison of Velocity Contours from Simulation with Full IP, Hybrid, and Full NS Domains.

\section{CONCLUDING REMARKS}

We have proposed a hybrid approach by coupling the DSMC-IP method and a Navier-Stokes solver for micro scale gas flows. The particle domain and the continuum domain exchanged information in every time step. The interface was adaptively determined so that the maximum benefits from hybrid schemes can be obtained. A condition similar to the Marshak condition was proposed to generate particles from the continuum side, and this 
strategy was applied to the simulation of a Couette flow. In another strategy of generating particles from the continuum side, buffer cells and reservoir cells were used, and this strategy was adopted in our general hybrid scheme. Simulation of a flow over a flat plate showed the ability of the hybrid approach and revealed the effects of the cutoff value of the continuum breakdown parameter B.
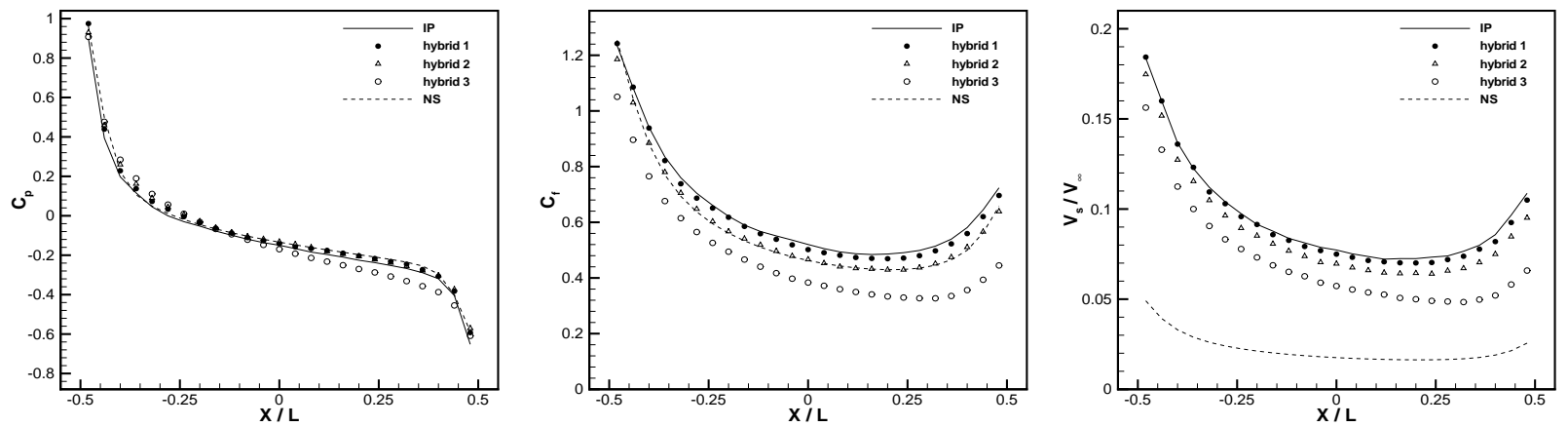

FIGURE 7. Comparison of Surface Properties from Simulation with Full IP, Hybrid, and Full NS Domains.

\section{ACKNOWLEDGMENTS}

The authors gratefully acknowledge the support for this work from the Air Force Office of Scientific Research through MURI grant F49620-98-1-0433.

\section{REFERENCES}

1. Karniadakis, G.E., and Beskok, A., Micro Flows: Fundamentals and Simulation, Springer, New York, 2001.

2. Senturia S.D., Microsystem Design, Kluwer Academic Publishers, Norwell, 2000.

3. Bird, G.A., Molecular Gas Dynamics and the Direct Simulation of Gas Flows, Oxford University Press, New York, 1994.

4. Hash, D.B., and Hassan, H.A., AIAA paper 96-0353 (1996).

5. Lumpkin, F.E., III, Stuart, P.C., and LeBeau, G.J., AIAA paper 96-1877 (1996).

6. Bourgat, J.F., Tallec, P.L., and Tidriri, M.D., Journal of Computational Physics, 127, 227-245 (1996).

7. Oh, C.K., and Oran, E.S., AIAA paper 98-0849 (1998).

8. Wadsworth, D.C., and Erwin, D.A., AIAA paper 90-1690 (1990).

9. Wadsworth, D.C., and Erwin, D.A., AIAA paper 92-2975 (1992).

10. Hash, D.B., and Hassan, H.A., Journal of Thermophysics and Heat Transfer, 10(2), 242-249 (1996).

11. Hash, D.B., and Hassan, H.A., AIAA paper 97-2507 (1997).

12. Tallec, P.L., and Mallinger F., Journal of Computational Physics, 136, $51-67$ (1997).

13. Alder, B., Physica A, 240, 193-195 (1997).

14. Flekkøy, E.G., Wagner, G., and Feder, J., Europhysics Letters, 52(3), 271-276 (2000).

15. Hadjiconstantinou, N.G., Journal of Computational physics, 154, 245-265 (1999).

16. Aktas, O., and Aluru, N.R., Journal of Computational Physics, 178, 342-372 (2002).

17. Roveda, R., Goldstein, D.B., and Varghese, P.L., Journal of Spacecraft and Rockets, 35(4), 258-265 (1998).

18. Roveda, R., Goldstein, D.B., and Varghese, P.L., Journal of Spacecraft and Rockets, 37(6), 753-760 (2000).

19. Tiwari, S., and Klar, A., Journal of Computational and Applied Mathematics, 90, 223-237 (1998).

20. Garcia, A.L., Bell, J.B., Crutchfield, W.Y., and Alder, B.J., Journal of Computational Physics, 154, 134-155 (1999).

21. Fan, J., and Shen, C., Journal of Computational Physics, 167, 393-412 (2001).

22. Sun, Q., and Boyd, I.D., Journal of Computational Physics, 179, 400-425 (2002).

23. Hirsch, C., Numerical Computational of Internal and External Flows I \& II, 1990.

24. Nance, R.P., Hash, D.B., and Hassan, H.A., Journal of Thermophyscics and Heat Transfer, 12(3), 447-449 (1998).

25. Guo, K., and Liaw, G.S., AIAA paper 2001-2953 (2001).

26. MacCormack, R.W., and Candler, G.V., Computers and Fluids, 17(1), 135-150 (1989).

27. Dietrich, S., and Boyd, I.D., Journal of Computational Physics, 126, 328-342 (1996).

28. Garcia, A.L., and Alder, B.J., Journal of Computational Physics, 140, 66-70 (1998).

29. Bird, G.A., AIAA Journal, 8(11), 1998-2003 (1970).

30. Boyd, I.D., Chen, G., and Candler, G.V., Physics of Fluids, 7(1), 210-219 (1995).

31. Tiwari, S., Journal of Computational Physics, 144, 710-726 (1998).

32. Gombosi, T.I., Gaskinetic Theory, Cambridge University Press, Cambridge, 1994. 